\title{
Non-Stochastic Private Function Evaluation
}

\author{
Farhad Farokhi and Girish Nair
}

\begin{abstract}
We consider private function evaluation to provide query responses based on private data of multiple untrusted entities in such a way that each cannot learn something substantially new about the data of others. First, we introduce perfect non-stochastic privacy in a two-party scenario. Perfect privacy amounts to conditional unrelatedness of the query response and the private uncertain variable of other individuals conditioned on the uncertain variable of a given entity. We show that perfect privacy can be achieved for queries that are functions of the common uncertain variable, a generalization of the common random variable. We compute the closest approximation of the queries that do not take this form. To provide a trade-off between privacy and utility, we relax the notion of perfect privacy. We define almost perfect privacy and show that this new definition equates to using conditional disassociation instead of conditional unrelatedness in the definition of perfect privacy. Then, we generalize the definitions to multi-party function evaluation (more than two data entities). We prove that uniform quantization of query responses, where the quantization resolution is a function of privacy budget and sensitivity of the query (cf., differential privacy), achieves function evaluation privacy.
\end{abstract}

Index Terms-Non-Stochastic Privacy; Common Uncertain Variable; Information Leakage; Quantization.

\section{INTRODUCTION}

Privacy research in information theory [1], [2] and computer science [3], [4] often deals with the problem of reporting privacy-preserving query responses based on private data available on a secure server. That is, when computing the privacypreserving responses, the server has access to the entire private dataset and generates a noisy output with desired utility and privacy against a third-party adversary. Those studies fail to investigate private information leakage from the query responses to individuals whose data is used for responding to the query, which amounts to privacy analysis in the presence of side-channel information. This is an important problem when multiple untrusted parties must come together to compute the response to an aggregate query.

In this work, we consider providing query responses based on the data of multiple untrusted entities in such a way that they cannot learn something substantially new about the data of others. We refer to this as private function evaluation. At first, we introduce and investigate perfect privacy in a twoparty scenario. This is the non-stochastic counterpart of perfect privacy in stochastic literature [5]-[7]. Perfect privacy equates to conditional unrelatedness of the query response and the private uncertain variable of other individuals conditioned on the uncertain variable of a given entity. We show that perfect privacy can be achieved for queries that are functions of the

The authors are with the Department of Electrical and Electronic Engineering at the University of Melbourne. e-mails: $\{$ ffarokhi,gnair $\} @$ unimelb.edu.au

The work of F. Farokhi is supported by an startup grant at Melbourne School of Engineering at the University of Melbourne. The work of G. Nair was supported by the Australian Research Council grant FT140100527. common uncertain variable, a generalization of the common random variable in the sense of [8]. For queries that do not take this form, we can approximate the query to compute the response with the most utility, i.e., closest worst-case response. To provide a trade-off between privacy and utility, we relax the notion of privacy. We define almost perfect nonstochastic privacy and show that this new definition equates to using conditional disassociation, borrowed from [9], instead of conditional unrelatedness in the definition of perfect privacy. We investigate the family of functions that can achieve almost perfect privacy. Then, we generalize our definition to multi-party function evaluation (more than two data entities). We prove that private function evaluation can be achieved by uniform quantization of the query responses, where the quantization resolution is a function of privacy budget and sensitivity of the query (cf., scale of the Laplace mechanism differential privacy [4]). We also investigate the utility of these reporting policies to establish utility-privacy trade-off.

Private function evaluation, at first glance, might seem related to private computation [10]-[12] and private information retrieval [13], [14]. However, in private function evaluation, the objective is not to hide the function to be computed (cf., private computation) or the datasets on which the function is evaluated (cf., private information retrieval). We are rather interested in ensuring that the parties contributing to the private dataset cannot infer the private information of the other parties. This links the problem more intimately to privacy-preserving distributed decision making [15]-[18]. However, all those papers consider stochastic mechanisms for ensuring privacy while, in this paper, we are interested in a non-stochastic notion of information leakage and privacy.

Despite their shortcomings, due to heuristic-based development, non-stochastic privacy-preserving policies have remained popular [19]-[21]. Those studies are motivated by concerns about the use of randomization in popular stochastic approaches. For instance, randomized policies, stemming from differential privacy in financial auditing, can potentially complicate fraud detection [22], [23] and cause difficulties in medical, health, or social research [24], [25]. This has motivated the use of information-theoretic tools to investigate non-stochastic privacy recently [26]-[29]. Nonetheless, those studies do not consider the private function computation setup in this paper.

\section{UNCERTAIN VARIABLES}

In this section, we present necessary preliminaries from nonstochastic information theory from [27], [30].

A sample space $\Omega$ models the set of uncertainties. An uncertain variable is a mapping from the sample space to a set of interest, such as $X: \Omega \rightarrow \mathbb{X}$. Here, $X(\omega)$ denotes a 
realization of uncertain variable $X$ corresponding to sample $\omega \in \Omega$. If $\mathbb{X}$ is finite, the uncertain variable $X$ is discrete. In this paper, we focus on discrete uncertain variables. The range of an uncertain variable $X$ is the set of all its realizations, e.g., $\llbracket X \rrbracket:=\{X(\omega): \omega \in \Omega\} \subseteq \mathbb{X}$. The joint range of any two uncertain variables $X: \Omega \rightarrow \mathbb{X}$ and $Y: \Omega \rightarrow \mathbb{Y}$ is $\llbracket X, Y \rrbracket:=\{(X(\omega), Y(\omega)): \omega \in \Omega\} \subseteq \mathbb{X} \times \mathbb{Y}$. The conditional range of uncertain variable $X$, conditioned on a realization of another uncertain variable $Y(\omega)=y$, is $\llbracket X \mid Y(\omega)=y \rrbracket:=\{X(\omega): \exists \omega \in \Omega$ such that $Y(\omega)=y\}=$ $X\left(Y^{-1}(y)\right) \subseteq \llbracket X \rrbracket$, where $Y^{-1}$ denotes the pre-image or inverse image of $Y$. Whenever it is evident from the context, $\llbracket X \mid y \rrbracket$ is used instead of $\llbracket X \mid Y(\omega)=y \rrbracket$ for the sake of brevity.

Uncertain variables $X_{1}, \ldots, X_{n}$ are unrelated if $\llbracket X_{1}, \ldots, X_{n} \rrbracket=\llbracket X_{1} \rrbracket \times \cdots \times \llbracket X_{n} \rrbracket$. Further, they are conditionally unrelated (conditioned on observations of $Y$ ) if $\llbracket X_{1}, \ldots, X_{n}\left|Y(\omega)=y \rrbracket=\llbracket X_{1}\right| Y(\omega)=y \rrbracket \times \cdots \times$ $\llbracket X_{n} \mid Y(\omega)=y \rrbracket$ for all $y \in \llbracket Y \rrbracket$. For two uncertain variables, this definition is equivalent to stating that $X_{1}$ and $X_{2}$ are unrelated if $\llbracket X_{1} \mid X_{2}(\omega)=x_{2} \rrbracket=\llbracket X_{1} \rrbracket, \forall x_{2} \in \llbracket X_{2} \rrbracket$, and vice versa [30]. Again, for two uncertain variables, $X_{1}$ and $X_{2}$ are conditionally unrelated (conditioned on realizations of $Y$ ) if $\llbracket X_{1}\left|X_{2}(\omega)=x_{2}, Y(\omega)=y \rrbracket=\llbracket X_{1}\right| Y(\omega)=y \rrbracket, \forall\left(x_{2}, y\right) \in$ $\llbracket X_{2}, Y \rrbracket[30]$.

The non-stochastic entropy of uncertain variable $X$ is

$$
H_{0}(X):=\log _{2}(|\llbracket X \rrbracket|)
$$

This is commonly referred to as the Hartley entropy [30], [31] and coincides with the Rényi entropy of order 0 for discrete variables, see, e.g., [32, Definition 1] and [33]. Conditional (or relative) entropy of uncertain variable $X$ given uncertain variable $Y$ is

$$
H_{0}(X \mid Y):=\max _{y \in \llbracket Y \rrbracket} \log _{2}(|\llbracket X| Y(\omega)=y \rrbracket \mid) .
$$

This coincides with the Arimoto-Rényi conditional entropy of order 0, see, e.g., [32, Definition 3] and [34]. So, the nonstochastic information between two uncertain variables $X$ and $Y$ can be defined as the difference of the entropy of $X$ with and without access to realizations of $Y$ :

$$
\begin{aligned}
I_{0}(X ; Y): & =H_{0}(X)-H_{0}(X \mid Y) \\
& =\min _{y \in \llbracket Y \rrbracket} \log _{2}\left(\frac{|\llbracket X \rrbracket|}{|\llbracket X| y \rrbracket \mid}\right) .
\end{aligned}
$$

Zeroth order information, defined above, is not symmetric, i.e., $I_{0}(X ; Y) \neq I_{0}(Y ; X)$, in general. This notion of information is also related to Kolmogorov's information gain $|\llbracket X \rrbracket| /|\llbracket X| y \rrbracket \mid$ and the 'combinatorial' conditional entropy $\log _{2}(|\llbracket X| y \rrbracket \mid)$ [35]. However, the combinatorial conditional entropy and the information gain are defined for a given realization $Y(\omega)=y$ while the non-stochastic information is for the worst-case realization.

In [27], it was observed that, in the context of informationtheoretic privacy, $I_{0}$ is not an appropriate measure of information leakage. This is because $I_{0}$ focuses on least informative observations while a privacy-intrusive adversary is interested in most informative realizations. Therefore, in [27], an alternative non-stochastic information leakage is proposed as

$$
L_{0}(X ; Y):=\max _{y \in \llbracket Y \rrbracket} \log _{2}\left(\frac{|\llbracket X \rrbracket|}{|\llbracket X| y \rrbracket \mid}\right) .
$$

Non-stochastic information leakage $L_{0}(X ; Y)$ captures the worst-case reduction in the complexity of brute-force guessing $X$ after observing $Y$ [36]. In general, $I_{0}$ and non-stochastic information leakage $L_{0}$ are not equal, i.e., $I_{0}(X ; Y) \neq$ $L_{0}(Y ; X)$. In fact, it is evident that $I_{0}(X ; Y) \leq L_{0}(X ; Y)$. Again, $L_{0}(X ; Y)$ is not symmetric. Note that $L_{0}(X ; Y) \geq 0$ with equality achieved if and only if $X$ and $Y$ are unrelated. Finally, we can similarly define conditional non-stochastic information leakage:

$$
\begin{aligned}
& L_{0}(X ; Y \mid Z) \\
& \quad:=\max _{(y, z) \in \llbracket Y, Z \rrbracket} \log _{2}\left(\frac{|\llbracket X| Z(\omega)=z \rrbracket \mid}{|\llbracket X| Y(\omega)=y, Z(\omega)=z \rrbracket \mid}\right) .
\end{aligned}
$$

Note that $L_{0}(Y ; X \mid Z) \geq 0$ with equality achieved if and only if $X$ and $Y$ are unrelated conditioned on $Z$.

In [30], maximin or non-stochastic information is introduced as a symmetric measure of information and its relationship with zero-error capacity is explored. To present the definition of the maximin information, we need to introduce overlap partitions.

\section{Definition 1 (Overlap Partition).}

- $x, x^{\prime} \in \llbracket X \rrbracket$ are overlap connected (via $\left.\llbracket X \mid Y \rrbracket\right), x \leftrightarrow$ $x^{\prime}$, if there exists a finite sequence of conditional ranges $\left\{\llbracket X \mid y_{i} \rrbracket\right\}_{i=1}^{n}$ such that $x \in \llbracket X\left|y_{1} \rrbracket, x^{\prime} \in \llbracket X\right| y_{n} \rrbracket$, and $\llbracket X\left|y_{i} \rrbracket \cap \llbracket X\right| y_{i+1} \rrbracket \neq \emptyset$ for all $i=1, \ldots, n-1$;

- $\mathcal{A} \subseteq \llbracket X \rrbracket$ is overlap connected if all $x, x^{\prime} \in \mathcal{A}$ are overlap connected;

- $\mathcal{A}, \mathcal{B} \subseteq \llbracket X \rrbracket$ are overlap isolated if there do not exist points $x \in \mathcal{A}$ and $x^{\prime} \in \mathcal{B}$ such that $x \leftrightarrow x^{\prime}$;

- An overlap partition of $\llbracket X \rrbracket$ is a set of sets $\llbracket X \mid Y \rrbracket \star=$ $\left\{\mathcal{A}_{i}\right\}_{i=1}^{n}$ such that $\llbracket X \rrbracket \subseteq \bigcup_{i=1}^{n} \mathcal{A}_{i}, \mathcal{A}_{i}, \mathcal{A}_{j}$ are overlap isolated if $j \neq i$, and $\mathcal{A}_{i}$ is overlap connected;

There always exists a unique overlap partition [30]. The maximin information is

$$
I_{\star}(X ; Y):=\log _{2}\left(|\llbracket X| Y \rrbracket_{\star} \mid\right) .
$$

Note that $I_{\star}(X ; Y) \geq 0$ and $I_{\star}(X ; Y)=0$ if and only if uncertain variables $X$ and $Y$ are unrelated.

Definition 2 (Taxicab Connectivity).

- $(x, y),\left(x^{\prime}, y^{\prime}\right) \in \llbracket X, Y \rrbracket$ are taxicab connected if there exists a sequence of points $\left\{\left(x_{i}, y_{i}\right)\right\}_{i=1}^{n} \subseteq \llbracket X, Y \rrbracket$ such that $\left(x_{1}, y_{1}\right)=(x, y),\left(x_{n}, y_{n}\right)=\left(x^{\prime}, y^{\prime}\right)$, and either $x_{i}=x_{i-1}$ or $y_{i}=y_{i-1}$ for all $i \in\{2, \ldots, n\}$;

- $\mathcal{A} \subseteq \llbracket X, Y \rrbracket$ is taxicab connected if all points in $\llbracket X, Y \rrbracket$ are taxicab connected;

- $\mathcal{A}, \mathcal{B} \subseteq \llbracket X, Y \rrbracket$ are taxicab isolated if there do not exist points $(x, y) \in \mathcal{A}$ and $\left(x^{\prime}, y^{\prime}\right) \in \mathcal{B}$ such that $(x, y)$ and $\left(x^{\prime}, y^{\prime}\right)$ are taxicab connected;

- A taxicab partition of $\llbracket X, Y \rrbracket$ is a set of sets $\mathfrak{T}(X ; Y):=$ $\left\{\mathcal{A}_{i}\right\}_{i=1}^{n}$ such that $\llbracket X, Y \rrbracket \subseteq \bigcup_{i=1}^{n} \mathcal{A}_{i}$, any $\mathcal{A}_{i}, \mathcal{A}_{j}$ are taxicab isolated if $j \neq i$, and $\mathcal{A}_{i}$ is taxicab connected. 
Again,there exists a unique taxicab partition $\mathfrak{T}(X ; Y)$ [30]. Furthermore, $\llbracket X \mid Y \rrbracket_{\star}$ are $\llbracket Y \mid X \rrbracket_{\star}$ are projections of the unique taxicab partition $\mathfrak{T}(X ; Y)$. Hence, $|\llbracket X| Y \rrbracket_{\star} \mid=$ $|\mathfrak{T}(X ; Y)|=|\llbracket Y| X \rrbracket_{\star} \mid$ implying that the maximin information is symmetric, i.e., $I_{\star}(X ; Y)=I_{\star}(Y ; X)$. The maximin information is related to the non-stochastic information leakage $I_{\star}(X ; Y) \leq L_{0}(X ; Y)$ [26], [37]. Due to symmetry of maximin information, $I_{\star}(X ; Y)=I_{\star}(Y ; X) \leq L_{0}(Y ; X)$.

\section{COMmon UnCERTAin VARIABle, INFORMATion, AND PERFECT PRIVACY}

In this section, we first discuss extension of common random variables in [8] to uncertain variables in line with the approach of [30], [38] for developing privacy-preserving policies. This extends the use of common information [39], also known as the Gács-Körner common information [40], in perfect privacy [41] to the non-stochastic framework.

\section{A. Common Uncertain Variable and Information}

We start by introducing the notion of common uncertain variables and relating it to overlap partitions and maximin information.

Definition 3 (Common Uncertain Variable). Let $X_{1}$ and $X_{2}$ be any two uncertain variables with disjoin 11 ranges.

- $\mathcal{G}$ is a bipartite graph with the vertex set $\mathcal{V}=\llbracket X_{1} \rrbracket \cup \llbracket X_{2} \rrbracket$ and the edge set $\mathcal{E}=\llbracket X_{1}, X_{2} \rrbracket$;

- $f_{1}: \llbracket X_{1} \rrbracket \rightarrow 2^{\llbracket X_{1} \rrbracket \cup \llbracket X_{2} \rrbracket}$ is a function that maps $x_{1} \in$ $\llbracket X_{1} \rrbracket \subseteq \mathcal{V}$ to the set of vertices in $\llbracket X_{1} \rrbracket \cup \llbracket X_{2} \rrbracket$ that are in the connected component of $\mathcal{G}$ containing $x_{1}$;

- $f_{2}: \llbracket X_{2} \rrbracket \rightarrow 2^{\llbracket X_{1} \rrbracket \cup \llbracket X_{2} \rrbracket}$ is a function that maps $x_{2} \in$ $\llbracket X_{2} \rrbracket \subseteq \mathcal{V}$ to the set of vertices in $\llbracket X_{1} \rrbracket \cup \llbracket X_{2} \rrbracket$ that are in the connected component of $\mathcal{G}$ containing $x_{2}$.

The common uncertain variable is $X_{1} \wedge X_{2}=f_{1} \circ X_{1}=$ $f_{2} \circ X_{2}$.

Similar to [8], we should note that the common uncertain variable $X_{1} \wedge X_{2}$ is the "largest" uncertain variable that can be extracted from uncertain variables $X_{1}$ and $X_{2}$.

Proposition 1. Assume that uncertain variables $X_{1}, X_{2}$, and $C$ exist such that $C=\bar{f}_{1} \circ X_{1}=\bar{f}_{2} \circ X_{2}$ for functions $\bar{f}_{1}: \llbracket X_{1} \rrbracket \rightarrow \llbracket C \rrbracket$ and $\bar{f}_{1}: \llbracket X_{2} \rrbracket \rightarrow \llbracket C \rrbracket$. There exists $g: 2^{\llbracket X_{1} \rrbracket \cup \llbracket X_{2} \rrbracket} \rightarrow \llbracket C \rrbracket$ such that $C=g\left(X_{1} \wedge X_{2}\right)$.

Proof: The proof follows the same line of reasoning as in the proof of Lemma 1 in [8].

For the unique overlap partition of $\llbracket X_{1} \rrbracket, \llbracket X_{1} \mid X_{2} \rrbracket_{\star}=$ $\left\{\mathcal{A}_{i}\right\}_{i=1}^{n_{1}}$ with $n_{1} \in \mathbb{N}$, define $\mathfrak{i}_{1}: \llbracket X_{1} \rrbracket \rightarrow\left\{1, \ldots, n_{1}\right\}$ such that $\mathfrak{i}_{1}\left(x_{1}\right)=i$ for which $x_{1} \in \mathcal{A}_{i}$. Similarly, for the unique overlap partition of $\llbracket X_{2} \rrbracket, \llbracket X_{2} \mid X_{1} \rrbracket_{\star}=\left\{\mathcal{B}_{i}\right\}_{i=1}^{n_{2}}$ with $n_{2} \in \mathbb{N}$, define $\mathfrak{i}_{2}: \llbracket X_{2} \rrbracket \rightarrow\left\{1, \ldots, n_{2}\right\}$ such that $\mathfrak{i}_{2}\left(x_{2}\right)=i$ for which $x_{2} \in \mathcal{B}_{i}$. The mappings $\mathfrak{i}_{1}$ and $\mathfrak{i}_{1}$ are well-defined because $\llbracket X_{1} \mid X_{2} \rrbracket_{\star}$ and $\llbracket X_{2} \mid X_{1} \rrbracket_{\star}$ are partitions.

\footnotetext{
${ }^{1}$ The disjoint assumption is just to simplify definition of the bipartite graph by making vertexes associated with alphabet of $X_{1}$ and $X_{2}$ distinguishable This is clearly without loss of generality as changing the event sets/alphabets of uncertain variables does not change their properties.
}

Definition 4 (Equivalence). Two uncertain variables $X$ and $Y$ are equivalent, $X \equiv Y$, if there exists a one-to-one correspondence $\rrbracket: \llbracket X \rrbracket \rightarrow \llbracket Y \rrbracket$ such that $Y=f \circ X$.

The notion of "equivalence" between two uncertain variables is weaker than that of "equality". If two uncertain variables are equal, they are equivalent as well. However, if two uncertain variables are equivalent, they could differ in terms of their range and hence not being equal to each other. This concept is explored for random variables in [42]. Note that entropy remains invariant under the equivalence relationship.

Proposition 2. $H_{0}(X)=H_{0}(Y)$ if $X \equiv Y$.

Proof. Since $X \equiv Y$, there must exist a one-to-one correspondence $f$ such that $Y=f \circ X$. Note that $|\llbracket Y \rrbracket|=\mid\{Y(\omega): \omega \in$ $\Omega\}|=|\{f(X(\omega)): \omega \in \Omega\}|=|\{X(\omega): \omega \in \Omega\}|=| \llbracket X \rrbracket \mid$, where the third equality follows from that $f$ is a one-to-one correspondence.

Proposition 3. $X_{1} \wedge X_{2} \equiv \mathfrak{i}_{1} \circ X_{1} \equiv \mathfrak{i}_{2} \circ X_{2}$ and $I_{\star}\left(X_{1} ; X_{2}\right)=$ $H_{0}\left(X_{1} \wedge X_{2}\right)$

Proof: Again, $\llbracket X_{1} \mid X_{2} \rrbracket_{\star}$ are $\llbracket X_{2} \mid X_{1} \rrbracket_{\star}$ are projections of the unique taxicab partition $\mathfrak{T}(X ; Y)$ [30]. The elements of the taxicab partition correspond to the connected components of the bipartite graph $\mathcal{G}$ [30], [38]. The rest of the proof follows from that entropy is invariant under equivalence relationship between uncertain variables (similar to the case of random variables in [42], [43]).

Remark 1 (Common Knowledge vs Common Information). Under additional measurability assumptions [38], taxicab partition and maximin information also relate to common knowledge in economics [44], [45] and logic [46], [47], not to be mistaken with common information [8], [39], [40], [48]. Instead of the graph theoretic definition for common information in [8] (and its extension to uncertain variables in Definition [3), common knowledge of a statement within a group refers to that everyone knows the validity of the aforementioned statement, everyone knows that everyone knows this, everyone knows that everyone knows that everyone knows this, and so on ad infinitum. This is modelled and captured using knowledge operators and knowledge field [44].

Remark 2 (Extension to Multi-Variables). To define common uncertain variable between multiple (more than two) uncertain variables, the approach of [49] for multi-variable extension of the Gács-Körner common information can be used. Let $X_{1} \ldots, X_{n}$ be any $n \geq 2$ uncertain variables with disjoint ranges. The common uncertain variable is recursively constructed by $X_{1} \wedge X_{2} \wedge \cdots \wedge X_{i-1} \wedge X_{i}=\left(X_{1} \wedge X_{2} \wedge\right.$ $\left.\cdots \wedge X_{i-1}\right) \wedge X_{i}$ for all $i=2, \ldots, n$. Note that the binary operation $\wedge$ between uncertain variables is associative [8] and commutative (see [42], [43] for the random variable case).

\footnotetext{
${ }^{2}$ The partitions of sample space $\Omega$ induced by $X$ and $Y$ are the same though their labeling may be different.
} 


\section{B. Perfect Privacy}

Perfect privacy [5], defined by adapting Shannon's perfect secrecy [50] to the privacy framework, states that an observations is perfectly private if it is statistically independent of the secret/private random variable. This concept has been recently further investigated [6], [7] as it provides a fundamental understanding of utility-privacy trade-off. In the non-stochastic case, independence can be replaced with unrelatedness. We can tailor this definition to the case of private function computation by assuming that, conditioned on the realization of the uncertain variable of each party, the outcome should not leak any information about the uncertain variable of the other party, i.e., the outcome should be conditionally unrelated to each uncertain variable.

Definition 5 (Perfect Privacy in Two-Party Function Evaluation). Let $X_{1}$ and $X_{2}$ be any two uncertain variables. The mapping $f: \llbracket X_{1}, X_{2} \rrbracket \rightarrow \mathbb{R}^{m}$ provides perfect privacy if $f\left(X_{1}, X_{2}\right)$ is unrelated to $X_{1}$ conditioned on $X_{2}$ and $f\left(X_{1}, X_{2}\right)$ is unrelated to $X_{2}$ conditioned on $X_{1}$.

Note that Definition 5 implies that the mapping $f$ provides perfect privacy if $\llbracket X_{2} \mid X_{1}(\omega)=x_{1}, Z(\omega)=z \rrbracket=$ $\llbracket X_{2} \mid X_{1}(\omega)=x_{1} \rrbracket$ and $\llbracket X_{1} \mid X_{2}(\omega)=x_{2}, Z(\omega)=z \rrbracket=$ $\llbracket X_{1} \mid X_{2}(\omega)=x_{2} \rrbracket$ with $Z=f\left(X_{1}, X_{2}\right)$. Perfect privacy for two-party function evaluation can be equivalently defined using conditional non-stochastic information leakage. This is proved in the next proposition.

Proposition 4. Mapping $f$ provides perfect privacy if and only if $L_{0}\left(f\left(X_{1}, X_{2}\right) ; X_{1} \mid X_{2}\right)=L_{0}\left(f\left(X_{1}, X_{2}\right) ; X_{2} \mid X_{1}\right)=0$.

Proof. We first prove that $f\left(X_{1}, X_{2}\right)$ is unrelated to $X_{1}$ conditioned on $X_{2}$ if and only if $L_{0}\left(f\left(X_{1}, X_{2}\right) ; X_{1} \mid X_{2}\right)=0$. First, if $f\left(X_{1}, X_{2}\right)$ is unrelated to $X_{1}$ conditioned on $X_{2}$, we get $\llbracket f\left(X_{1}, X_{2}\right) \mid X_{1}(\omega)=x_{1}, X_{2}(\omega)=x_{2} \rrbracket=$ $\llbracket f\left(X_{1}, X_{2}\right) \mid X_{2}(\omega)=x_{2} \rrbracket$ for all $\left(x_{1}, x_{2}\right) \in \llbracket X_{1}, X_{2} \rrbracket$. Thus, $\left|\llbracket f\left(X_{1}, X_{2}\right)\right| X_{1}(\omega)=x_{1}, X_{2}(\omega)=x_{2} \rrbracket \mid=$ $\left|\llbracket f\left(X_{1}, X_{2}\right)\right| X_{2}(\omega)=x_{2} \rrbracket \mid$ for all $\left(x_{1}, x_{2}\right) \in \llbracket X_{1}, X_{2} \rrbracket$. Therefore, definition of conditional non-stochastic information leakage in (5) implies that $L_{0}\left(f\left(X_{1}, X_{2}\right) ; X_{1} \mid X_{2}\right)=0$. Now, we prove the reverse. Assume that $L_{0}\left(f\left(X_{1}, X_{2}\right) ; X_{1} \mid X_{2}\right)=$ 0 . Then, it must be that $\left|\llbracket f\left(X_{1}, X_{2}\right)\right| X_{1}(\omega)=$ $x_{1}, X_{2}(\omega)=x_{2} \rrbracket|=| \llbracket f\left(X_{1}, X_{2}\right)\left|X_{2}(\omega)=x_{2} \rrbracket\right|$ for all $\left(x_{1}, x_{2}\right) \in \llbracket X_{1}, X_{2} \rrbracket$. Note that $\llbracket f\left(X_{1}, X_{2}\right) \mid X_{1}(\omega)=$ $x_{1}, X_{2}(\omega)=x_{2} \rrbracket \subseteq \llbracket f\left(X_{1}, X_{2}\right) \mid X_{2}(\omega)=x_{2} \rrbracket$. Therefore, $\left|\llbracket f\left(X_{1}, X_{2}\right)\right| X_{1}(\omega)=x_{1}, X_{2}(\omega)=x_{2} \rrbracket \mid=$ $\left|\llbracket f\left(X_{1}, X_{2}\right)\right| X_{2}(\omega)=x_{2} \rrbracket \mid$ implies that $\llbracket f\left(X_{1}, X_{2}\right) \mid X_{1}(\omega)=$ $x_{1}, X_{2}(\omega)=x_{2} \rrbracket=\llbracket f\left(X_{1}, X_{2}\right) \mid X_{2}(\omega)=x_{2} \rrbracket$. This shows that $f\left(X_{1}, X_{2}\right)$ is unrelated to $X_{1}$ conditioned on $X_{2}$. Similarly, we can prove that $f\left(X_{1}, X_{2}\right)$ is unrelated to $X_{2}$ conditioned on $X_{1}$ if and only if $L_{0}\left(f\left(X_{1}, X_{2}\right) ; X_{2} \mid X_{1}\right)=0$. This concludes the proof.

Evaluating common uncertain variable $X_{1} \wedge X_{2}$ and its functions provide perfect privacy. This is proved in the following proposition.

Proposition 5. The following statements hold:

- $X_{1} \wedge X_{2}$ is unrelated to $X_{1}$ conditioned on $X_{2}$;
- $X_{1} \wedge X_{2}$ is unrelated to $X_{2}$ conditioned on $X_{1}$.

Proof: Note that because $X_{1} \wedge X_{2}=\mathfrak{i}_{2} \circ X_{2}$, we have $\llbracket X_{1} \wedge X_{2}\left|X_{1}(\omega)=x_{1}, X_{2}(\omega)=x_{2} \rrbracket=\llbracket i_{2}\left(X_{2}\right)\right| X_{1}(\omega)=$ $x_{1}, X_{2}(\omega)=x_{2} \rrbracket=\left\{\mathfrak{i}_{2}\left(x_{2}\right)\right\}=\llbracket \mathfrak{i}_{2}\left(X_{2}\right) \mid X_{2}(\omega)=x_{2} \rrbracket$. Therefore, $X_{1} \wedge X_{2}$ is unrelated to $X_{1}$ conditioned on $X_{2}$. The proof for the other case is similar.

As stated earlier, all functions of the common uncertain variable also provide perfect privacy. The inverse is however also true. In fact, any function that provides perfect privacy must only be computable based on the common uncertain variable. This is explored in the following proposition.

Proposition 6. For any $f: \llbracket X_{1}, X_{2} \rrbracket \rightarrow \mathbb{R}^{m}$ providing perfect privacy, there exists $g: 2^{\llbracket X_{1} \rrbracket \cup \llbracket X_{2} \rrbracket} \rightarrow \mathbb{R}^{m}$ such that $f\left(X_{1}, X_{2}\right)=g\left(X_{1} \wedge X_{2}\right)$.

Proof: Because $f$ is a noiseless deterministic mapping, $\llbracket f\left(X_{1}, X_{2}\right) \mid X_{1}(\omega)=x_{1}, X_{2}(\omega)=x_{2} \rrbracket$ is a singleton for all $\left(x_{1}, x_{2}\right) \in \llbracket X_{1}, X_{2} \rrbracket$. Noting that $f\left(X_{1}, X_{2}\right)$ is unrelated to $X_{1}$ conditioned on $X_{2}$, $\llbracket f\left(X_{1}, X_{2}\right) \mid X_{1}(\omega)=x_{1}, X_{2}(\omega)=$ $x_{2} \rrbracket=\llbracket f\left(X_{1}, X_{2}\right) \mid X_{2}(\omega)=x_{2} \rrbracket$ for all $\left(x_{1}, x_{2}\right) \in \llbracket X_{1}, X_{2} \rrbracket$. As a result, $\llbracket f\left(X_{1}, X_{2}\right) \mid X_{2}(\omega)=x_{2} \rrbracket$ is also a singleton for all $\left(x_{1}, x_{2}\right) \in \llbracket X_{1}, X_{2} \rrbracket$. Therefore, there exists $g_{2}$ such that $f\left(X_{1}, X_{2}\right)=g_{2} \circ X_{2}$. Similarly, there exists $g_{1}$ such that $f\left(X_{1}, X_{2}\right)=g_{1} \circ X_{1}$. Therefore, $g_{1} \circ X_{1}=f\left(X_{1}, X_{2}\right)=$ $g_{2} \circ X_{2}$. By Proposition 11, there must exists $g$ such that $f\left(X_{1}, X_{2}\right)=g\left(X_{1} \wedge X_{2}\right)$.

Not all functions provides perfect privacy. This is evident as not all functions can be rewritten in terms of the common variable $X_{1} \wedge X_{2}$. For instance, $f\left(X_{1}, X_{2}\right)=X_{1}$ cannot be written in terms of the common uncertain variable. This function also does not provide perfect privacy. Thus, we might need to approximate such a function with one that provides perfect privacy. This can be done by

$$
\begin{array}{ll}
\min _{f^{\prime}} & \max _{\left(x_{1}, x_{2}\right) \in \llbracket X_{1}, X_{2} \rrbracket}\left\|f\left(x_{1}, x_{2}\right)-f^{\prime}\left(x_{1}, x_{2}\right)\right\|, \\
\text { s.t. } & f^{\prime} \text { provides perfect privacy. }
\end{array}
$$

If $f^{\prime}$ provides perfect privacy, it must be in the form of $g\left(X_{1} \wedge\right.$ $X_{2}$ ) for some mapping $g$. Therefore, in light of Proposition 3 , we have

$$
\begin{aligned}
\max _{\left(x_{1}, x_{2}\right) \in \llbracket X_{1}, X_{2} \rrbracket}\left\|f\left(x_{1}, x_{2}\right)-f^{\prime}\left(x_{1}, x_{2}\right)\right\| \\
=\max _{i} \max _{\left(x_{1}, x_{2}\right) \in \mathcal{A}_{i}}\left\|f\left(x_{1}, x_{2}\right)-f^{\prime}\left(x_{1}, x_{2}\right)\right\|,
\end{aligned}
$$

where $\mathfrak{T}\left(X_{1} ; X_{2}\right)=\left\{\mathcal{A}_{i}\right\}_{i=1}^{\left|\mathfrak{T}\left(X_{1} ; X_{2}\right)\right|}$. The solution of (7) is given by

$$
f^{\prime}\left(x_{1}, x_{2}\right)=\operatorname{center}\left(f\left(\mathcal{A}_{i}\right)\right), \quad\left(x_{1}, x_{2}\right) \in \mathcal{A}_{i},
$$

where, for any set $\mathcal{A} \subseteq \mathbb{R}^{m}$,

$$
\operatorname{center}(\mathcal{A}):=\underset{z^{\prime} \in \operatorname{conv}(\mathcal{A})}{\arg \min } \max _{z \in \mathcal{A}}\left\|z^{\prime}-z\right\|,
$$

with $\operatorname{conv}(\mathcal{A})$ denoting the convex hull of $\mathcal{A}$. In general, the condition for perfect privacy can be strong. It also does not offer a systematic way for trading-off utility and privacy. In the remainder of this paper, we relax this notion of privacy. 


\section{Almost Perfect Privacy}

We can relax the conditional unrelatedness in the definition of perfect privacy in Definition 5 to get a weaker notion of privacy. Proposition 4 shows that $\max \left\{L_{0}\left(f\left(X_{1}, X_{2}\right) ; X_{1} \mid X_{2}\right), L_{0}\left(f\left(X_{1}, X_{2}\right) ; X_{2} \mid X_{1}\right)\right\}=0$ if and only if mapping $f$ provides perfect privacy. By definition, $\max \left\{L_{0}\left(f\left(X_{1}, X_{2}\right) ; X_{1} \mid X_{2}\right), L_{0}\left(f\left(X_{1}, X_{2}\right) ; X_{2} \mid X_{1}\right)\right\} \geq 0$. Therefore, we can relax perfect privacy by requiring that $\max \left\{L_{0}\left(f\left(X_{1}, X_{2}\right) ; X_{1} \mid X_{2}\right), L_{0}\left(f\left(X_{1}, X_{2}\right) ; X_{2} \mid X_{1}\right)\right\}$ is small rather than zero.

Definition 6 ( $\gamma$-Privacy in Two-Party Function Evaluation). Let $X_{1}$ and $X_{2}$ be any two uncertain variables. For $\gamma \geq$ 0 , mapping $f: \llbracket X_{1}, X_{2} \rrbracket \rightarrow \mathbb{R}^{m}$ provides $\gamma$-privacy if $\max \left\{L_{0}\left(f\left(X_{1}, X_{2}\right) ; X_{1} \mid X_{2}\right), L_{0}\left(f\left(X_{1}, X_{2}\right) ; X_{2} \mid X_{1}\right)\right\} \leq \gamma$.

In what follows, we borrow disassociation from [9] as a relaxation of unrelatedness. This way, we can investigate $\gamma$ privacy in more depth by casting it in terms of disassociation rather than conditional information leakage.

Definition 7 (Disassociated Uncertain Variables). For $\delta \in$ $[0,1]$, two uncertain variables $X$ and $Y$ are $\delta$-disassociated if

$$
\begin{gathered}
\frac{|\llbracket X| Y(\omega)=y_{1} \rrbracket \cap \llbracket X\left|Y(\omega)=y_{2} \rrbracket\right|}{|\llbracket X \rrbracket|} \geq \delta, \\
\forall y_{1}, y_{2} \in \llbracket Y \rrbracket: y_{1} \neq y_{2}, \\
\frac{|\llbracket Y| X(\omega)=x_{1} \rrbracket \cap \llbracket Y\left|X(\omega)=x_{2} \rrbracket\right|}{|\llbracket Y \rrbracket|} \geq \delta, \\
\forall x_{1}, x_{2} \in \llbracket X \rrbracket: x_{1} \neq x_{2} .
\end{gathered}
$$

If only (8a) holds, $X$ is partially $\delta$-disassociated with $Y$.

Proposition 7. Two uncertain variables $X$ and $Y$ are unrelated if and only if they are 1-disassociated.

Proof. The proof is outlined in [9]. For the proof of the sufficiency, note that $|\llbracket X| Y(\omega)=y_{1} \rrbracket \cap \llbracket X\left|Y(\omega)=y_{2} \rrbracket\right|=$ $|\llbracket X \rrbracket|$ for all $y_{1}, y_{2} \in \llbracket Y \rrbracket$ if $\delta=1$ in (8a). Noting that, $\llbracket X\left|Y(\omega)=y_{1} \rrbracket \cap \llbracket X\right| Y(\omega)=y_{2} \rrbracket \subseteq \llbracket X \rrbracket,|\llbracket X| Y(\omega)=$ $y_{1} \rrbracket \cap \llbracket X\left|Y(\omega)=y_{2} \rrbracket\right|=|\llbracket X \rrbracket|$ implies that $\llbracket X \mid Y(\omega)=$ $y_{1} \rrbracket \cap \llbracket X \mid Y(\omega)=y_{2} \rrbracket=\llbracket X \rrbracket$. And, in turn, this implies that $\llbracket X\left|Y(\omega)=y_{1} \rrbracket=\llbracket X\right| Y(\omega)=y_{2} \rrbracket=\llbracket X \rrbracket$ Similarly, $\llbracket Y\left|X(\omega)=x_{1} \rrbracket=\llbracket Y\right| X(\omega)=x_{2} \rrbracket=\llbracket Y \rrbracket$ for all $x_{1}, x_{2} \in \llbracket X \rrbracket$ if $\delta=1$ in (8b). Therefore, $X$ and $Y$ must be unrelated. The proof of the necessity is similar and follows from straightforward algebraic manipulations.

As $\delta$ increases, any two $\delta$-disassociated uncertain variables "appear more unrelated" and, as demonstrated in Proposition 7, 1-disassociated implies unrelatedness between two uncertain variables. Therefore, we can think of $\delta$-disassociation as a relaxation of the notion of unrelatedness.

Proposition 8. The following inequalities hold for $\delta$ disassociated uncertain variables $X$ and $Y$ :

- $L_{0}(X ; Y) \leq-\log _{2}(\delta)$;

- $L_{0}(Y ; X) \leq-\log _{2}(\delta)$;

- $I_{\star}(X ; Y) \leq-\log _{2}(\delta)$.

Proof. Let us prove that $L_{0}(X ; Y) \leq-\log _{2}(\delta)$. If $|\llbracket Y \rrbracket|=1$, $L_{0}(X ; Y)=0$ and the inequality trivially holds. Therefore, without loss of generality, we concentrate on $|\llbracket Y \rrbracket|>1$. For any $y \in \llbracket Y \rrbracket$ and $y^{\prime} \neq y$, we get $|\llbracket X| Y(\omega)=y \rrbracket \mid \geq$ $|\llbracket X| Y(\omega)=y \rrbracket \cap \llbracket X\left|Y(\omega)=y^{\prime} \rrbracket\right| \geq \delta|\llbracket X \rrbracket|$. Therefore, $|\llbracket X \rrbracket| /|\llbracket X| Y(\omega)=y \rrbracket \mid \leq 1 / \delta$. The proof for $L_{0}(Y ; X) \leq$ $-\log _{2}(\delta)$ follows the same line of reasoning. Finally, the proof can be concluded by noting that $I_{\star}(X ; Y) \leq L_{0}(X ; Y)$ [26], [37].

Proposition 8 shows that the information content between any two $\delta$-disassociated uncertain variables $X$ and $Y$ reduces as $\delta$ gets larger. In the limit for $\delta=1, L_{0}(X ; Y)=$ $L_{0}(Y ; X)=I_{\star}(X ; Y)=0$. This also shows that $X$ and $Y$ are unrelated if they are 1-disassociated.

Definition 8 (Conditionally Disassociated Uncertain Variables). For $\delta \in[0,1]$, two uncertain variables $X$ and $Y$ are $\delta$-disassociated conditioned on uncertain variable $Z$ if

$$
\begin{gathered}
\frac{|\llbracket X| Y(\omega)=y_{1}, Z(\omega)=z \rrbracket \cap \llbracket X\left|Y(\omega)=y_{2}, Z(\omega)=z \rrbracket\right|}{|\llbracket X| Z(\omega)=z \rrbracket \mid} \geq \delta, \\
\forall y_{1}, y_{2} \in \llbracket Y \mid Z(\omega)=z \rrbracket: y_{1} \neq y_{2}, \forall z \in \llbracket Z \rrbracket, \quad \\
\frac{|\llbracket Y| X(\omega)=x_{1}, Z(\omega)=z \rrbracket \cap \llbracket Y\left|X(\omega)=x_{2}, Z(\omega)=z \rrbracket\right|}{|\llbracket Y| Z(\omega)=z \rrbracket \mid} \geq \delta, \\
\forall x_{1}, x_{2} \in \llbracket X \mid Z(\omega)=z \rrbracket: x_{1} \neq x_{2}, \forall z \in \llbracket Z \rrbracket . \quad
\end{gathered}
$$

If only (9a) holds, $X$ is partially $\delta$-disassociated with $Y$ conditioned on $Z$.

Following the same argument as Proposition 7, two uncertain variables $X$ and $Y$ are unrelated conditioned on uncertain variable $Z$ if they are 1 -disassociated conditioned on $Z$. Hence, we can think of conditional disassociation as a relaxation of conditional unrelatedness.

Proposition 9. Assume that two uncertain variables $X$ and $Y$ are $\delta$-disassociated conditioned on uncertain variable $Z$ for some $\delta \in[0,1]$. Then, $L_{0}(X ; Y \mid Z) \leq-\log _{2}(\delta)$.

Proof. For any $(y, z) \in \llbracket Y, Z \rrbracket$ such that $|\llbracket Y| Z(\omega)=z \rrbracket \mid>1$, we get $|\llbracket X| Y(\omega)=y, Z(\omega)=z \rrbracket|\geq| \llbracket X \mid Y(\omega)=y, Z(\omega)=$ $z \rrbracket \cap \llbracket X\left|Y(\omega)=y^{\prime}, Z(\omega)=z \rrbracket\right| \geq \delta|\llbracket X| Z(\omega)=z \rrbracket \mid$. Therefore, $|\llbracket X| Z(\omega)=z \rrbracket|/| \llbracket X|Y(\omega)=y, Z(\omega)=z \rrbracket| \leq 1 / \delta$. For any $(y, z) \in \llbracket Y, Z \rrbracket$ such that $|\llbracket Y| Z(\omega)=z \rrbracket \mid=1$, we also get $|\llbracket X| Y(\omega)=y, Z(\omega)=z \rrbracket \mid=1$ because $\llbracket X|Y(\omega)=y, Z(\omega)=z \rrbracket \subseteq \llbracket Y| Z(\omega)=z \rrbracket$. Therefore, $|\llbracket X| Z(\omega)=z \rrbracket|/| \llbracket X|Y(\omega)=y, Z(\omega)=z \rrbracket|=1 \leq 1 / \delta$. This implies that $L_{0}(X ; Y \mid Z) \leq-\log _{2}(\delta)$.

Proposition 10. Let $X_{1}$ and $X_{2}$ be any two uncertain variables. For $\gamma \geq 0$, mapping $f: \llbracket X_{1}, X_{2} \rrbracket \rightarrow \mathbb{R}^{m}$ provides $\gamma$-privacy if $f\left(X_{1}, X_{2}\right)$ and $X_{1}$ are $e^{-\gamma}$-disassociated conditioned on $X_{2}$, and $f\left(X_{1}, X_{2}\right)$ and $X_{2}$ are $e^{-\gamma}$-disassociated conditioned on $X_{1}$.

Proof. The proof follows form the application of Proposition 9

Proposition 10 shows that we can relax the definition of perfect privacy by requiring conditional disassociation instead of conditional unrelatedness.

Definition 9 ( $\delta$-Overlap Connectivity). For $\delta \in[0,1]$, 
- $x, x^{\prime} \in \llbracket X \rrbracket$ are $\delta$-overlap connected (via $\left.\llbracket X \mid Y \rrbracket\right), x \leftrightarrow \delta$ $x^{\prime}$, if there exists a sequence of points $\left\{y_{i}\right\}_{i=1}^{n} \subseteq \llbracket Y \rrbracket$ such that $x \in \llbracket X\left|Y(\omega)=y_{1} \rrbracket, x^{\prime} \in \llbracket X\right| Y(\omega)=y_{n} \rrbracket$, and $|\llbracket X| Y(\omega)=y_{i} \rrbracket \cap \llbracket X\left|Y(\omega)=y_{i-1} \rrbracket\right| /|\llbracket X \rrbracket| \geq \delta$, for all $i \in\{2, \ldots, n\}$;

- if $x, x^{\prime} \in \llbracket X \rrbracket$ are $\delta$-overlap connected with $n=1$, they are singly $\delta$-overlap connected;

- $\mathcal{A} \subseteq \llbracket X \rrbracket$ is (singly) $\delta$-overlap connected if all points in $\llbracket X \rrbracket$ are (singly) $\delta$-taxicab connected;

- $\mathcal{A}, \mathcal{B} \subseteq \llbracket X \rrbracket$ are $\delta$-overlap isolated if there do not exist points $x \in \mathcal{A}$ and $x^{\prime} \in \mathcal{B}$ such that $x$ m $\delta x^{\prime}$;

- A $\delta$-overlap partition of $\llbracket X \rrbracket$ is a set of sets $\llbracket X \mid Y \rrbracket_{\delta}:=$ $\left\{\mathcal{A}_{i}\right\}_{i=1}^{n}$ such that $\llbracket X \rrbracket \subseteq \bigcup_{i=1}^{n} \mathcal{A}_{i}, \mathcal{A}_{i}, \mathcal{A}_{j}$ are $\delta$-overlap isolated if $j \neq i$, and $\mathcal{A}_{i}$ is $\delta$-overlap connected;

- A $\delta$-overlap family of $\llbracket X \rrbracket$, denoted by $\llbracket X \mid Y \rrbracket_{\star}^{\delta}$, is the largest $\delta$-overlap partition of $\llbracket X \rrbracket$ such that each set in the family contains a singly $\delta$-overlap connected set of the form $\llbracket X \mid Y(\omega)=y \rrbracket$, there exists a set containing any two singly $\delta$-overlap connected points, and the measure of overlap between any two sets in the family is at most $\delta|\llbracket X \rrbracket|$.

For any two uncertain variables $X$ and $Y$, there always exists a $\delta$-overlap family of $\llbracket X \rrbracket$ while the uniqueness is guaranteed if $X$ and $Y$ are $\delta$-disassociated [9, Theorems 3-4]. For a given $\delta$-overlap family of $\llbracket X_{1} \rrbracket, \llbracket X_{1} \mid X_{2} \rrbracket_{\star}^{\delta}=\left\{\mathcal{A}_{i}\right\}_{i=1}^{n_{1}}$ with $n_{1} \in \mathbb{N}$, define $\mathfrak{i}_{1}^{\delta}: \llbracket X_{1} \rrbracket \rightarrow\left\{1, \ldots, n_{1}\right\}$ such that $\mathfrak{i}_{1}^{\delta}\left(x_{1}\right)=i$ for which $x_{1} \in \mathcal{A}_{i}$. Similarly, for a given $\delta$ overlap family of $\llbracket X_{2} \rrbracket, \llbracket X_{2} \mid X_{1} \rrbracket_{\star}^{\delta}=\left\{\mathcal{B}_{i}\right\}_{i=1}^{n_{2}}$ with $n_{2} \in \mathbb{N}$, define $\mathfrak{i}_{2}^{\delta}: \llbracket X_{2} \rrbracket \rightarrow\left\{1, \ldots, n_{2}\right\}$ such that $\mathfrak{i}_{2}^{\delta}\left(x_{2}\right)=i$ for which $x_{2} \in \mathcal{B}_{i}$. The mappings $\mathfrak{i}_{1}^{\delta}$ and $\mathfrak{i}_{1}^{\delta}$ are well-defined because $\llbracket X_{1} \mid X_{2} \rrbracket_{\star}^{\delta}$ and $\llbracket X_{2} \mid X_{1} \rrbracket_{\star}^{\delta}$ partition $\llbracket X_{1} \rrbracket$ and $\llbracket X_{2} \rrbracket$, respectively.

Proposition 11. If $X_{1}$ and $X_{2}$ are $\delta$-disassociated for $\delta \in$ $[0,1], \mathfrak{i}_{1}^{\delta} \circ X \equiv \mathfrak{i}_{2}^{\delta} \circ X_{2}$.

Proof. Note that, if $X_{1}$ and $X_{2}$ are $\delta$-disassociated, $\llbracket X_{1} \mid X_{2} \rrbracket_{\star}^{\delta}$ are $\llbracket X_{2} \mid X_{1} \rrbracket_{\star}^{\delta}$ are unique [9. Theorems 3-4] and are projections of the unique $\delta$-taxicab family of $\llbracket X, Y \rrbracket$ denoted by $\llbracket X, Y \rrbracket_{\star}^{\delta}[9$, Theorem 5]. Therefore, there is a bijection from $\llbracket X_{1} \mid X_{2} \rrbracket_{\star}^{\delta}$ to $\llbracket X, Y \rrbracket_{\star}^{\delta}$ and another bijection from $\llbracket X, Y \rrbracket_{\star}^{\delta}$ to $\llbracket X_{1} \mid X_{2} \rrbracket_{\star}^{\delta}$.

Proposition 12. For $\delta \in[0,1]$, the following statements hold:

- $\mathfrak{i}_{1}^{\delta} \circ X$ and $Y$ are $\delta$-disassociated conditioned on $X$;

- $\mathfrak{i}_{2}^{\delta} \circ Y$ and $X$ are $\delta$-disassociated conditioned on $Y$.

Proof. The proof is by reductio ad absurdum. Assume that $\mathfrak{i}_{1}^{\delta} \circ X$ and $Y$ are not $\delta$-disassociated conditioned on $X$. One of the following cases can occur.

Case 1: There exist $x \in \llbracket X \rrbracket$ and $y_{1}, y_{2} \in \llbracket Y \mid X(\omega)=x \rrbracket$ such that $y_{1} \neq y_{2}$ and $\left|\llbracket \mathfrak{i}_{1}^{\delta} \circ X\right| Y(\omega)=y_{1}, X(\omega)=x \rrbracket \cap$ $\llbracket \mathfrak{i}_{1}^{\delta} \circ X\left|Y(\omega)=y_{2}, X(\omega)=x \rrbracket\right| /\left|\llbracket \mathfrak{i}_{1}^{\delta} \circ X\right| X(\omega)=x \rrbracket \mid<\delta$. Note that $\left|\llbracket i_{1}^{\delta} \circ X\right| X(\omega)=x \rrbracket \mid=1$ and $\llbracket i_{1}^{\delta} \circ X \mid Y(\omega)=$ $y_{1}, X(\omega)=x \rrbracket=\llbracket \mathfrak{i}_{1}^{\delta} \circ X \mid Y(\omega)=y_{2}, X(\omega)=x \rrbracket=\llbracket i_{1}^{\delta} \circ$ $X \mid X(\omega)=x \rrbracket$. This results in contradiction as it gives $\mid \llbracket i_{1}^{\delta} \circ$ $X\left|Y(\omega)=y_{1}, X(\omega)=x \rrbracket \cap \llbracket i_{1}^{\delta} \circ X\right| Y(\omega)=y_{2}, X(\omega)=$ $x \rrbracket|/| \llbracket \mathfrak{i}_{1}^{\delta} \circ X|X(\omega)=x \rrbracket|=1$ while $\delta \leq 1$.
Case 2: There exist $x \in \llbracket X \rrbracket$ and $z_{1}, z_{2} \in \llbracket \mathfrak{i}_{1}^{\delta} \circ X \mid X(\omega)=$ $x \rrbracket$ such that $z_{1} \neq z_{2}$ and $|\llbracket Y| \mathfrak{i}_{1}^{\delta} \circ X(\omega)=z_{1}, X(\omega)=x \rrbracket \cap$ $\llbracket Y\left|\mathfrak{i}_{1}^{\delta} \circ X(\omega)=z_{2}, X(\omega)=x \rrbracket\right| /|\llbracket Y| X(\omega)=x \rrbracket \mid<\delta$. This is however not possible as $\llbracket \mathfrak{i}_{1}^{\delta} \circ X \mid X(\omega)=x \rrbracket$ is a singleton so it cannot contain two distinct points $z_{1}, z_{2}$.

The proof for showing that $\mathfrak{i}_{2}^{\delta} \circ Y$ and $X$ are $\delta$-disassociated conditioned on $Y$ follows the same line of reasoning.

Propositions 11 and 12 show that equivalent uncertain variables $\mathfrak{i}_{1}^{\delta} \circ X$ and $\mathfrak{i}_{2}^{\delta} \circ X_{2}$ are akin to relaxations of the common uncertain variable (c.f., Proposition 3).

Corollary 13. Let $f: \llbracket X_{1}, X_{2} \rrbracket \rightarrow \mathbb{R}^{m}$ be any mapping for which there exists $g_{1}: \llbracket i_{1}^{\delta} \circ X \rrbracket \rightarrow \mathbb{R}^{m}$ or $g_{2}: \llbracket i_{2}^{\delta} \circ Y \rrbracket \rightarrow \mathbb{R}^{m}$ such that $f\left(X_{1}, X_{2}\right)=g_{1} \circ \mathfrak{i}_{1}^{\delta} \circ X$ or $f\left(X_{1}, X_{2}\right)=g_{2} \circ \mathfrak{i}_{2}^{\delta} \circ Y$. Then, $f$ provides $\log _{2}(-\delta)$-privacy.

\section{Private Multi-Party Function Evaluation}

In this section, we generalize the results of the earlier sections to more than two entities. Consider $n \geq 2$ entities, each possessing an uncertain variable $X_{i}$. Define $X=\left(X_{i}\right)_{i=1}^{n}$. We are interested in publishing the result of evaluating a function $f: \llbracket X \rrbracket \rightarrow \llbracket Y \rrbracket$ on the realization of the uncertain variables possessed by those entities. We want this publication to be done in a privacy-preserving manner. We first generalize the notion of privacy from two-party function evaluations.

Definition 10 ( $\gamma$-Privacy in Multi-Party Function Evaluation). Let $X_{1}, X_{2}, \ldots, X_{n}$ be any $n \geq 1$ uncertain variables. For $\gamma \geq 0$, mapping $f: \llbracket X_{1}, \ldots, X_{n} \rrbracket \rightarrow \mathbb{R}^{m}$ provides $\gamma$-privacy if $\max _{1 \leq i \leq n} L_{0}\left(f\left(X_{1}, \ldots, X_{n}\right) ; X_{-i} \mid X_{i}\right) \leq \gamma$.

As earlier said, we might not be able to evaluate any function $f$ in a privacy-preserving manner. Therefore, we might need to approximate function $f$ with one that can be privately evaluated $f^{\prime}: \llbracket X \rrbracket \rightarrow \llbracket Z \rrbracket$, where $Z=f^{\prime} \circ X$. Doing so, we publish the result of evaluating another function $f^{\prime}$ instead $f$. The error in the function evaluation is measured by

$$
\mathcal{E}\left(f^{\prime}, f\right)=\sup _{x \in \llbracket X \rrbracket} \rho\left(f(x), f^{\prime}(x)\right),
$$

where $\rho: \llbracket Y \rrbracket \times \llbracket Z \rrbracket \rightarrow \mathbb{R}$ is a distance function. In what follows, we use $\rho(y, z)=\|y-z\|$ for $\llbracket Y \rrbracket, \llbracket Z \rrbracket \subseteq \mathbb{R}^{m}$.

Definition 11 (Accuracy). Any $f^{\prime}$ is said to be $\beta$-accurate for $\beta>0$ if $\mathcal{E}\left(f^{\prime}, f\right) \leq \beta$.

An important problem is to find $\gamma$-private mapping $f^{\prime}$, if one exists, that is a $\beta$-accurate approximation of a desired function $f$. We investigate this in the remainder of this paper.

Proposition 14. Let $f^{\prime}$ be such that

$$
\left|\llbracket f^{\prime}(X)\right| X_{i}(\omega)=x_{i} \rrbracket \mid \leq \epsilon+1, \forall\left(x_{i}, x_{-i}\right) \in \llbracket X \rrbracket, \forall i .
$$

Then, $f^{\prime}$ provides $\log _{2}(1+\epsilon)$-privacy.

Proof. Note that, since $f^{\prime}$ is a deterministic mapping, $\left|\llbracket f^{\prime}(X)\right| X_{i}(\omega)=x_{i}, X_{-i}(\omega)=x_{-i} \rrbracket \mid=1$. Therefore, if $\left|\llbracket f^{\prime}(X)\right| X_{i}(\omega)=x_{i} \rrbracket \mid \leq \epsilon+1$, we get $\left|\llbracket f^{\prime}(X)\right| X_{i}(\omega)=$ $x_{i} \rrbracket|/| \llbracket f^{\prime}(X)\left|X_{i}(\omega)=x_{i}, X_{-i}(\omega)=x_{-i} \rrbracket\right| \leq \epsilon+1$ for all $\left(x_{i}, x_{-i}\right) \in \llbracket X \rrbracket$ and all $i$. 
Linear quantizers can achieve privacy for most functions. These functions have been previously used to provide privacy in the sense of non-stochastic information leakage [27]. Let us first formalize linear quantizers.

Definition 12 (Linear Quantizer). A q-level quantizer $\mathcal{Q}$ : $\left[x_{\min }, x_{\max }\right] \rightarrow\left\{b_{1}, \ldots, b_{q}\right\}$ is a piecewise constant function defined as

$$
Q(x)=\left\{\begin{array}{lc}
b_{1}, & x \in\left[x_{1}, x_{2}\right), \\
b_{2}, & x \in\left[x_{2}, x_{3}\right), \\
\vdots & \vdots \\
b_{q-1}, & x \in\left[x_{q-1}, x_{q}\right), \\
b_{q}, & x \in\left[x_{q}, x_{q+1}\right],
\end{array}\right.
$$

where $\left(b_{i}\right)_{i=1}^{q}$ are distinct symbols and $x_{1} \leq x_{2} \leq \cdots \leq$ $x_{q}$ are real numbers such that $x_{1}=x_{\min }, x_{q+1}=x_{\max }$, $x_{i+1}-x_{i}=\left(x_{\max }-x_{\min }\right) / q$ for all $1 \leq i \leq q$. This is a mid-point linear quantizer if $b_{i}=\left(x_{i}+x_{i+1}\right) / 2$ for all $i$.

In the next theorem, we show that using linear quantizers provides a universal mechanism for ensuring $\epsilon$-privacy. This is particularly important as we do not need to develop problemdependent privacy-preserving policies.

Theorem 15. Assume that $f$ is Lipschitz continuous, i.e., there exists $L>0$ such that $\left|f(x)-f\left(x^{\prime}\right)\right| \leq L\left\|x-x^{\prime}\right\|_{\infty}$ for all $x, x^{\prime} \in \llbracket X \rrbracket$, and $\llbracket X_{i} \rrbracket \subseteq\left[x_{\min }, x_{\max }\right]$ for all $i$. Then, $f^{\prime}=\mathcal{M} \circ f$, where $\mathcal{M}$ is a $\lfloor\exp (\gamma)-1\rfloor$-level mid-point linear quantizer over $\llbracket f(X) \rrbracket$, is $\gamma$-private and $\beta$-accurate with $\beta \geq L\left(x_{\max }-x_{\min }\right) /\lfloor\exp (\gamma)-1\rfloor$.

Proof. Note that $\left|\llbracket \mathfrak{M} \circ f\left(\left\{x_{i}\right\} \times \llbracket X_{-i} \rrbracket\right) \rrbracket\right| \leq|\llbracket \mathfrak{M} \circ f(\llbracket X \rrbracket) \rrbracket| \leq$ $\lfloor\exp (\gamma)-1\rfloor \leq \exp (\gamma)-1$ because $\llbracket \mathfrak{M} \circ f\left(\left\{x_{i}\right\} \times \llbracket X_{-i} \rrbracket\right) \rrbracket \subseteq$ $\llbracket \mathfrak{M} \circ f(\llbracket X \rrbracket) \rrbracket$. In light of Proposition [14, this proves that $f^{\prime}=\mathcal{M} \circ f$ is $\gamma$-private. Due to Lipschitz continuity of $f$, $f(\llbracket X \rrbracket) \subseteq\left[y_{\min }, y_{\max }\right]$, where $y_{\max }-y_{\min } \leq L\left(x_{\max }-x_{\min }\right)$. Therefore, using $\lfloor\exp (\gamma)-1\rfloor$-level mid-point linear quantizer, $\left|f(x)-f^{\prime}(x)\right| \leq \beta$ with $\beta \geq L\left(x_{\max }-x_{\min }\right) /\lfloor\exp (\gamma)-1\rfloor$ for all $x, x^{\prime} \in \llbracket X \rrbracket$. This shows that $\mathcal{E}\left(f^{\prime}, f\right) \leq \beta$ with $\beta \geq$ $L\left(x_{\max }-x_{\min }\right) /\lfloor\exp (\gamma)-1\rfloor$.

Theorem 15 shows that private function evaluation can be achieved by uniform quantization of the query responses, where the quantization resolution is a function of privacy budget $\gamma$ and sensitivity of the query $L\left(x_{\max }-x_{\min }\right)$ (cf., scale of the Laplace mechanism differential privacy [4]). Note that $L\left(x_{\max }-x_{\min }\right)$ captures the sensitivity of $f$, i.e., how much the output of the function $f$ varies if one of its entries change. For the mechanism in Theorem 15 , we get

$$
\begin{aligned}
\beta \exp (\gamma) & \geq \beta(\exp (\gamma)-1) \\
& \geq L\left(x_{\max }-x_{\min }\right)(\exp (\gamma)-1) /\lfloor\exp (\gamma)-1\rfloor \\
& \geq L\left(x_{\max }-x_{\min }\right) .
\end{aligned}
$$

This inequality provides a utility-privacy trade-off for nonstochastic private function evaluation.

\section{CONCLusions}

We consider private function evaluation to provide query responses based on private data of multiple untrusted entities in such a way that no entity can learn something substantially new about the data of others. We prove that uniform quantization of the query responses achieves privacy.

\section{REFERENCES}

[1] F. du Pin Calmon and N. Fawaz, "Privacy against statistical inference," in 2012 50th annual Allerton conference on communication, control, and computing (Allerton), pp. 1401-1408, 2012.

[2] I. Issa, S. Kamath, and A. B. Wagner, "An operational measure of information leakage," in 2016 Annual Conference on Information Science and Systems (CISS), pp. 234-239, 2016.

[3] C. Dwork, F. McSherry, K. Nissim, and A. Smith, "Calibrating noise to sensitivity in private data analysis," in Theory of Cryptography Conference, pp. 265-284, 2006.

[4] C. Dwork, "Differential privacy: A survey of results," in Theory and Ap plications of Models of Computation (M. Agrawal, D. Du, Z. Duan, and A. Li, eds.), (Berlin, Heidelberg), pp. 1-19, Springer Berlin Heidelberg, 2008.

[5] G. Miklau and D. Suciu, "A formal analysis of information disclosure in data exchange," Journal of Computer and System Sciences, vol. 73, no. 3, pp. 507-534, 2007.

[6] F. P. Calmon, A. Makhdoumi, and M. Médard, "Fundamental limits of perfect privacy," in 2015 IEEE International Symposium on Information Theory (ISIT), pp. 1796-1800, 2015.

[7] B. Rassouli and D. Gunduz, "On perfect privacy," in 2018 IEEE International Symposium on Information Theory (ISIT), pp. 2551-2555, 2018.

[8] S. Wolf and J. Wultschleger, "Zero-error information and applications in cryptography," in Information Theory Workshop, pp. 1-6, 2004.

[9] A. Rangi and M. Franceschetti, "Towards a non-stochastic information theory," in 2019 IEEE International Symposium on Information Theory (ISIT), pp. 997-1001, IEEE, 2019.

[10] M. Kim and J. Lee, "Private secure coded computation," in 2019 IEEE International Symposium on Information Theory (ISIT), pp. 1097-1101, 2019.

[11] A. Heidarzadeh and A. Sprintson, "Private computation with side information: The single-server case," in 2019 IEEE International Symposium on Information Theory (ISIT), pp. 1657-1661, 2019.

[12] B. Tahmasebi and M. A. Maddah-Ali, "Private sequential function computation," in 2019 IEEE International Symposium on Information Theory (ISIT), pp. 1667-1671, 2019.

[13] H.-Y. Lin, S. Kumar, E. Rosnes, and A. G. i Amat, "Asymmetry helps: Improved private information retrieval protocols for distributed storage," in 2018 IEEE Information Theory Workshop (ITW), pp. 1-5, 2018.

[14] K. Banawan and S. Ulukus, "Private information retrieval from multiple access channels," in 2018 IEEE Information Theory Workshop (ITW), pp. 1-5, 2018.

[15] K. Chaudhuri and C. Monteleoni, "Privacy-preserving logistic regression," in Advances in Neural Information Processing Systems, pp. 289296, 2009.

[16] M. Abadi, A. Chu, I. Goodfellow, H. B. McMahan, I. Mironov, K. Talwar, and L. Zhang, "Deep learning with differential privacy," in Proceedings of the 2016 ACM SIGSAC Conference on Computer and Communications Security, pp. 308-318, 2016.

[17] N. Wu, F. Farokhi, D. Smith, and M. A. Kaafar, "The value of collaboration in convex machine learning with differential privacy," in IEEE Symposium on Security and Privacy (IEEE SP), 2020.

[18] M. J. Wainwright, M. I. Jordan, and J. C. Duchi, "Privacy aware learning," in Advances in Neural Information Processing Systems, pp. 14301438, 2012.

[19] P. Samarati, "Protecting respondents identities in microdata release," IEEE Transactions on Knowledge and Data Engineering, vol. 13, no. 6, pp. 1010-1027, 2001.

[20] L. Sweeney, "k-anonymity: A model for protecting privacy," International Journal of Uncertainty, Fuzziness and Knowledge-Based Systems, vol. 10, no. 05, pp. 557-570, 2002.

[21] A. Machanavajjhala, J. Gehrke, D. Kifer, and M. Venkitasubramaniam, " $\ell$-diversity: privacy beyond $k$-anonymity," in 22nd International Conference on Data Engineering (ICDE'06), pp. 24-24, 2006.

[22] R. Bhaskar, A. Bhowmick, V. Goyal, S. Laxman, and A. Thakurta, "Noiseless database privacy," in International Conference on the Theory and Application of Cryptology and Information Security, pp. 215-232, 2011. 
[23] S. U. Nabar, B. Marthi, K. Kenthapadi, N. Mishra, and R. Motwani, "Towards robustness in query auditing," in Proceedings of the 32nd international conference on Very large data bases, pp. 151-162, 2006.

[24] F. K. Dankar and K. El Emam, "Practicing differential privacy in health care: A review," Transactions on Data Privacy, vol. 6, no. 1, pp. 35-67, 2013.

[25] J. Mervis, "Researchers object to census privacy measure," vol. 363, no. 6423, pp. 114-114, 2019.

[26] F. Farokhi, "Noiseless privacy," arXiv preprint arXiv:1910.13027, 2019.

[27] F. Farokhi, "Development and analysis of deterministic privacypreserving policies using non-stochastic information theory," IEEE Transactions on Information Forensics and Security, vol. 14, no. 10, pp. 2567-2576, 2019.

[28] N. Ding and F. Farokhi, "Developing non-stochastic privacy-preserving policies using agglomerative clustering," IEEE Transactions on Information Forensics and Security, vol. 15, pp. 3911-3923, 2020.

[29] F. Farokhi, "Non-stochastic hypothesis testing for privacy," IET Information Security, August 2020.

[30] G. N. Nair, "A nonstochastic information theory for communication and state estimation," IEEE Transactions on Automatic Control, vol. 58, no. 6, pp. 1497-1510, 2013.

[31] R. V. L. Hartley, "Transmission of information," Bell System Technical Journal, vol. 7, no. 3, pp. 535-563, 1928.

[32] I. Sason and S. Verdú, "Arimoto-rényi conditional entropy and bayesian $m$-ary hypothesis testing," IEEE Transactions on Information Theory, vol. 64, no. 1, pp. 4-25, 2017.

[33] A. Rényi, "On measures of entropy and information," in Proceedings of the 4th Berkeley Symposium on Mathematical Statistics and Probability, Volume 1: Contributions to the Theory of Statistics, 1961.

[34] S. Arimoto, "Information measures and capacity of order $\alpha$ for discrete memoryless channels," in Proceedings of the 2nd Colloquium on Topics on Information Theory, Keszthely, Hungary, vol. 16, p. 1975.

[35] A. N. Kolmogorov and V. M. Tikhomirov, " $\varepsilon$-entropy and $\varepsilon$-capacity of sets in function spaces," Uspekhi Matematicheskikh Nauk, vol. 14, no. 2, pp. 3-86, 1959. English translation American Mathematical Society Translations, series 2, vol. 17, pp. 277-364.

[36] F. Farokhi and N. Ding, "Measuring information leakage in nonstochastic brute-force guessing," arXiv preprint arXiv:2004.10911, 2020.

[37] N. Ding and F. Farokhi, "Developing non-stochastic privacy-preserving policies using agglomerative clustering," IEEE Transactions on Information Forensics and Security, vol. 15, pp. 3911-3923, 2020.

[38] A. Mahajan, "On the relationship between maximin information and common knowledge," 2014. Technical Note, http://www.ece.mcgill.ca/ amahaj1/projects/information/preprint/maximin-information.pdf

[39] C. Shannon, "The lattice theory of information," Transactions of the IRE professional Group on Information Theory, vol. 1, no. 1, pp. 105-107, 1953.

[40] P. Gács and J. Körner, "Common information is far less than mutual information," Problems of Control and Information Theory, vol. 2, no. 2, pp. 149-162, 1973.

[41] S. Asoodeh, F. Alajaji, and T. Linder, "Notes on information-theoretic privacy," in 2014 52nd Annual Allerton Conference on Communication, Control, and Computing (Allerton), pp. 1272-1278, 2014.

[42] H. Li and E. K. Chong, "On a connection between information and group lattices," Entropy, vol. 13, no. 3, pp. 683-708, 2011.

[43] V. Griffith, E. K. Chong, R. G. James, C. J. Ellison, and J. P. Crutchfield, "Intersection information based on common randomness," Entropy, vol. 16, no. 4, pp. 1985-2000, 2014.

[44] R. J. Aumann, "Agreeing to disagree," The annals of statistics, pp. 12361239,1976

[45] R. J. Aumann, "Interactive epistemology I: Knowledge," International Journal of Game Theory, vol. 28, no. 3, pp. 263-300, 1999.

[46] J.-J. C. Meyer and W. Van Der Hoek, Epistemic logic for AI and computer science, vol. 41. Cambridge University Press, 2004.

[47] H. Van Ditmarsch, W. van Der Hoek, and B. Kooi, Dynamic epistemic logic, vol. 337. Springer Science \& Business Media, 2007.

[48] A. Wyner, "The common information of two dependent random variables," IEEE Transactions on Information Theory, vol. 21, no. 2, pp. 163-179, 1975.

[49] H. Tyagi, P. Narayan, and P. Gupta, "When is a function securely computable?," IEEE Transactions on Information Theory, vol. 57, no. 10, pp. 6337-6350, 2011.

[50] C. E. Shannon, "Communication theory of secrecy systems," Bell System Technical Journal, vol. 28, no. 4, pp. 656-715, 1949. 